\title{
The Exponentiated Marshall-Olkin Fréchet Distribution
}

\author{
Mahmoud M. Mansour \\ College of Business Administration, Yanbu Department of MIS \\ Taibah University, Saudi Arabia \\ Department of Statistics, Mathematics and Insurance \\ Benha University, Egypt \\ mmmansour@taibahu.edu.sa \\ Enayat M. Abd Elrazik \\ College of Business Administration, Yanbu Department of MIS, \\ Taibah University, Saudi Arabia \\ Department of Statistics, Mathematics and Insurance \\ Benha University, Egypt \\ ekhalilabelgawad@taibahu.edu.sa \\ Nadeem Shafique Butt \\ Department of Family and Community Medicine \\ King Abdul Aziz University, Jeddah, Kingdom of Saudi Arabia \\ nshafique@kau.edu.sa
}

\begin{abstract}
A new five-parameter model called the exponentiated Marshall-Olkin Fréchet distribution is studied. Various of its mathematical properties including ordinary and incomplete moments, quantile and generating functions and order statistics are investigated. The proposed density function can be expressed as a linear mixture of Fréchet densities. The maximum likelihood method is used to estimate the model parameters. The flexibility of the new distribution is proved empirically using two real data sets.
\end{abstract}

Keywords: Exponentiated Marshall-Olkin-G Family, Fréchet Distribution, Generating Function, Goodness-of-fit, Maximum Likelihood, Order Statistic

\section{Introduction}

The Fréchet distribution, also known as type II extreme value distribution, is one of the important distributions in extreme value theory and it has wide applicability in extreme value theory. This distribution was proposed by Maurice Fréchet (1924), who investigated it as one possible limit distribution for a sequence of maxima. The Fréchet distribution is widely used in applications involving stochastic phenomena such as rainfall, floods, air pollution (Kotz and Nadarajah, 2000), material properties in engineering applications (Harlow, 2002), analyzing wind speed data (Zaharim et al., 2009) and advanced mathematical results on point processes and regularly varying functions (Resnick, 2013), among others. Further details about the Fréchet distribution and its applications can be explored in Kotz and Nadarajah (2000).

The statistical literature contains many extended forms of the Fréchet distribution. For example, Nadarajah and Kotz (2003) pioneered the exponentiated Fréchet, Nadarajah and Gupta (2004) and Barreto-Souza et al. (2011) studied the beta Fréchet, Mahmoud and 
Mandouh (2013) proposed the transmuted Fréchet, Krishna et al. (2013) introduced the Marshall-Olkin Fréchet, Silva et al. (2013) defined the gamma extended Fréchet, Elbatal et al. (2014) studied the transmuted exponentiated Fréchet, Mead and Abd-Eltawab (2014) introduced the Kumaraswamy Fréchet, Afify et al. (2015) investigated the transmuted Marshall-Olkin Fréchet, Afify et al. (2016a) studied the Kumaraswamy Marshall-Olkin Fréchet, Afify et al. (2016b) proposed the Weibull Fréchet, Tablada and Cordeiro (2017) defined the modified Fréchet and Mead et al. (2017) proposed the beta exponential Fréchet distributions.

In this article, we define and study a new five-parameter model called the exponentiated Marshall-Olkin Fréchet (EMOFr) distribution and provide some of its properties. We prove, by means of two applications, that the EMOFr distributions can give better fits than most of the above mentioned distributions.

The new model is generated by applying the exponentiated Marshal-Olkin-G (EMO-G) family (Dias et al., 2016) to the Fréchet distribution. Dias et al. (2016) defined the EMO$\mathrm{G}$ family of continuous distributions with three extra shape parameters by cumulative distribution function (cdf)

$$
F(x)=\left\{\frac{1-[1-G(x)]^{b}}{1-p[1-G(x)]^{b}}\right\}^{a} .
$$

The probability density function (pdf) of the EMO-G is given by

$$
f(x)=a b(1-p) g(x)[1-G(x)]^{b-1} \frac{\left\{1-[1-G(x)]^{b}\right\}^{a-1}}{\left\{1-p[1-G(x)]^{b}\right\}^{a+1}}
$$

where $a>0, b>0$ and $p \in(-\infty, 1)$ are shape parameters.

The rest of this chapter is organized as follows. In Section 2, we define the EMOFr distribution, provide its special cases and some plots for its pdf and hazard rate function (hrf). In Section 3, we provide a useful mixture representation for its pdf. In Section 4, we derive some of its mathematical properties. Maximum likelihood estimation of the model parameters is addressed in Section 5. In Section 6, we provide two applications to real data to illustrate the importance and flexibility of the new distribution.

\section{The EMOFr Distribution}

In this section, we define the EMOFr distribution. The new model generated by applying the exponentiated Marshall-Olkin transformation to the Fréchet distribution.

The cdf of the Fréchet distribution is given by (for $x>0$ )

$$
G(x ; \alpha, \beta)=\mathrm{e}^{-\left(\frac{\alpha}{x}\right)^{\beta}} .
$$

The corresponding pdf is given by

$$
g(x ; \alpha, \beta)=\beta \alpha^{\beta} x^{-\beta-1} \mathrm{e}^{-\left(\frac{\alpha}{x}\right)^{\beta}},
$$

where $\alpha>0$ is a scale parameter and $\beta>0$ is a shape parameter, respectively. 
Now, we proceed to define the new EMOFr distribution. By inserting the cdf of the Fréchet distribution in equation (1), we obtain the cdf of the EMOFr

$$
F(x)=\left\{\frac{1-\left[1-\mathrm{e}^{-\left(\frac{\alpha}{x}\right)^{\beta}}\right]^{b}}{1-p\left[1-\mathrm{e}^{-\left(\frac{\alpha}{x}\right)^{\beta}}\right]^{b}}\right\}^{a}
$$

The corresponding pdf of the EMOFr is given by

$$
f(x)=a b(1-p) \beta \alpha^{\beta} x^{-\beta-1} \mathrm{e}^{-\left(\frac{\alpha}{x}\right)^{\beta}}\left[1-\mathrm{e}^{-\left(\frac{\alpha}{x}\right)^{\beta}}\right]^{b-1} \frac{\left\{1-\left[1-\mathrm{e}^{-\left(\frac{\alpha}{x}\right)^{\beta}}\right]^{b}\right\}^{a-1}}{\left\{1-p\left[1-\mathrm{e}^{-\left(\frac{\alpha}{x}\right)^{\beta}}\right]^{b}\right\}^{a+1}}
$$

where $a>0, b>0, p \in(-\infty, 1)$ and $\beta>0$ are shape parameters and $\alpha>0$ is a scale parameter. Henceforth, we denote by $X \sim \operatorname{EMOFr}(\alpha, \beta, a, b, p)$ a random variable having pdf (4).

The hrf of $X$ is given by

$$
h(x)=\frac{a b(1-p) \beta \alpha^{\beta} x^{-\beta-1} \mathrm{e}^{-\left(\frac{\alpha}{x}\right)^{\beta}}\left[1-\mathrm{e}^{-\left(\frac{\alpha}{x}\right)^{\beta}}\right]^{b-1}\left\{1-\left[1-\mathrm{e}^{-\left(\frac{\alpha}{x}\right)^{\beta}}\right]^{b}\right\}^{a-1}}{\left\{1-p\left[1-\mathrm{e}^{-\left(\frac{\alpha}{x}\right)^{\beta}}\right]^{b}\right\}^{a+1}-\left\{1-\left[1-\mathrm{e}^{\left.\left.-\left(\frac{\alpha}{x}\right)^{\beta}\right]^{b}\right\}^{a}\left\{1-p\left[1-\mathrm{e}^{-\left(\frac{\alpha}{x}\right)^{\beta}}\right]^{b}\right\}} .\right.\right.}
$$

Plots of the EMOFr pdf for some parameter values are displayed in Figure 1. Figure 2 displays some possible shapes of the hrf of the EMOFr model for selected parameter values.

The EMOFr is a flexible model which contains 15 special models are listed in Table 1.

\section{Linear Representation}

In this section, we derive a useful linear mixture representation for the cdf and pdf of the EMOFr distribution. The cdf of the EMOFr in (3) can be expressed as

$$
F(x)=\left\{1-\left[1-\mathrm{e}^{-\left(\frac{\alpha}{x}\right)^{\beta}}\right]^{b}\right\}^{a}\left\{1-p\left[1-\mathrm{e}^{-\left(\frac{\alpha}{x}\right)^{\beta}}\right]^{b}\right\}^{-a}
$$


Table 1: Special models of the EMOFr model

\begin{tabular}{|c|c|c|c|c|c|c|}
\hline$\alpha$ & $\beta$ & $a$ & $b$ & $p$ & Reduced Model & Author(s) \\
\hline$\alpha$ & $\beta$ & $a$ & $b$ & 0 & EG-Fr & Cordeiro et al. (2013) \\
\hline$\alpha$ & 2 & $a$ & $b$ & 0 & EG-IR & - \\
\hline$\alpha$ & 1 & $a$ & $b$ & 0 & EG-IE & Krishna et al. (2013) \\
\hline$\alpha$ & $\beta$ & 1 & $b$ & $p$ & MO-Fr & - \\
\hline$\alpha$ & 2 & 1 & $b$ & $p$ & MO-IR & \\
\hline$\alpha$ & 1 & 1 & $b$ & $p$ & MO-IE & \\
\hline$\alpha$ & $\beta$ & 1 & $b$ & 0 & PHR-Fr & \\
\hline$\alpha$ & 2 & 1 & $b$ & 0 & PHR-IR & Trayer (1964) \\
\hline$\alpha$ & 1 & 1 & $b$ & 0 & PHR-IE & \\
\hline$\alpha$ & $\beta$ & $a$ & 1 & 0 & PRHR-Fr & \\
\hline$\alpha$ & 2 & $a$ & 1 & 0 & PRHR-IR & \\
\hline$\alpha$ & 1 & $a$ & 1 & 0 & PRHR-IE & \\
\hline$\alpha$ & $\beta$ & 1 & 1 & 0 & Fr & IR \\
\hline$\alpha$ & 2 & 1 & 1 & 0 & & \\
\hline$\alpha$ & 1 & 1 & 1 & 0 & IE & \\
\hline
\end{tabular}

Abbreviations: $\mathrm{IR}=$ Inverse Rayleigh, $\mathrm{IE}=$ Inverse Exponential.
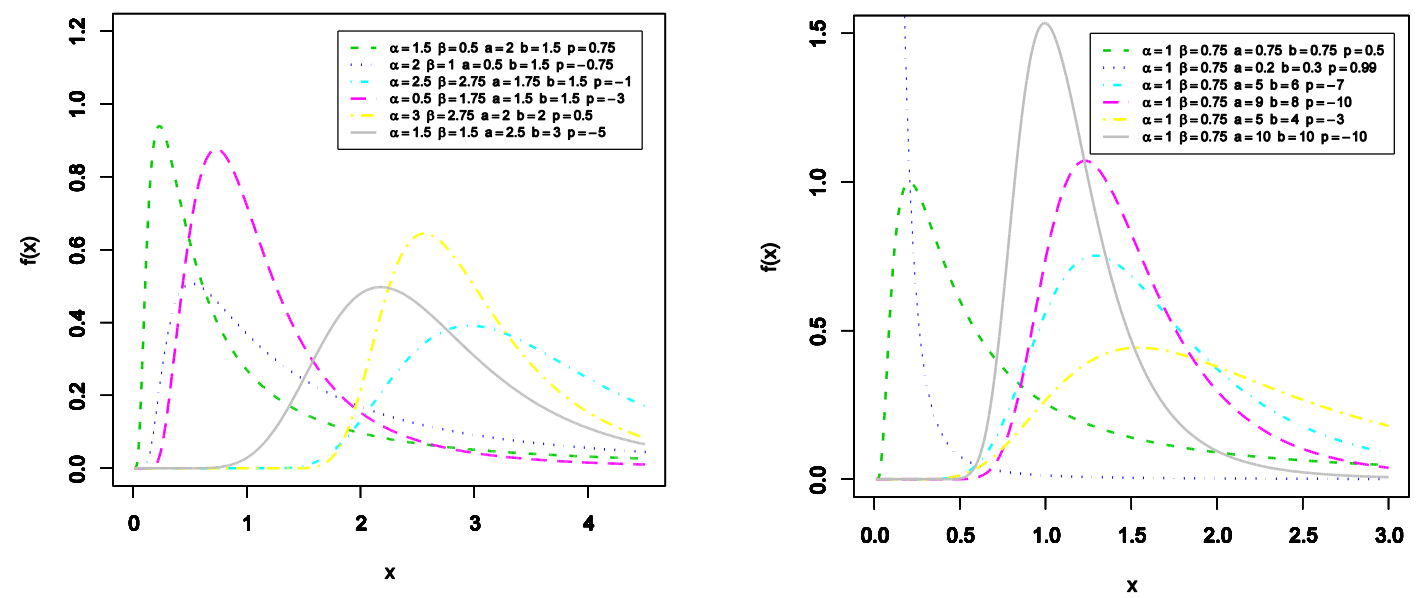

Figure 1: Plots of the EMOFr density function for selected parameter values 

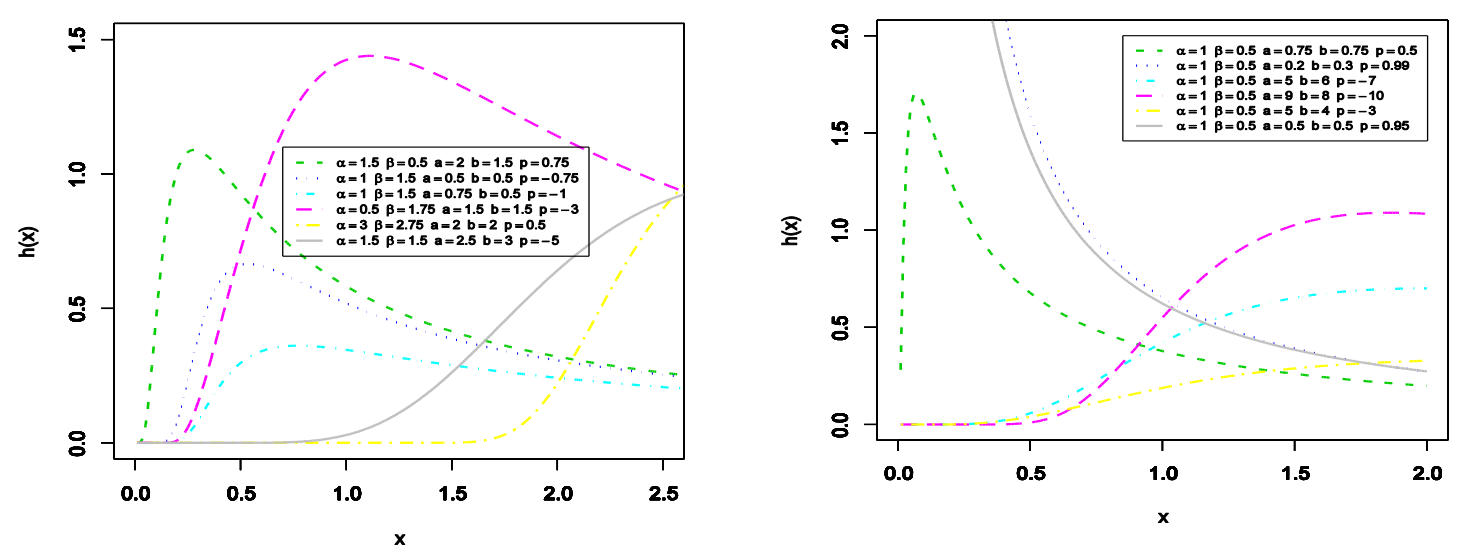

Figure 2: Plots of the EMOFr hrf for selected parameter values

Applying the binomial expansion defined by

$$
(1-z)^{-\varphi}=\sum_{i=0}^{\infty}(-1)^{i}\left(\begin{array}{l}
-\varphi \\
i
\end{array}\right) z^{i}
$$

Then, the cdf of the EMOFr reduces to

$$
F(x)={ }_{i=0}^{\infty}(-1)^{i} p^{i}\left(\begin{array}{l}
-a \\
i
\end{array}\right)\left[1-\mathrm{e}^{-\left(\frac{\alpha}{x}\right)^{\beta}}\right]^{b i}\left\{1-\left[1-\mathrm{e}^{-\left(\frac{\alpha}{x}\right)^{\beta}}\right]^{b}\right\}^{a} .
$$

Using the generalized binomial expansion defined by

$$
(1-z)^{\varphi}=\sum_{i=0}^{\infty}(-1)^{i}\left(\begin{array}{l}
\varphi \\
i
\end{array}\right) z^{i}
$$

we can write

$$
\left\{1-\left[1-\mathrm{e}^{-\left(\frac{\alpha}{x}\right)^{\beta}}\right]^{b}\right\}^{a}=\sum_{j=0}^{\infty}(-1)^{j}\left(\begin{array}{l}
a \\
j
\end{array}\right)\left[1-\mathrm{e}^{-\left(\frac{\alpha}{x}\right)^{\beta}}\right]^{b j}
$$

Then, equation (6) reduces to

$$
F(x)=\sum_{i, j=0}^{\infty}(-1)^{i+j} p^{i}\left(\begin{array}{c}
-a \\
i
\end{array}\right)\left(\begin{array}{l}
a \\
j
\end{array}\right)\left[1-\mathrm{e}^{-\left(\frac{\alpha}{x}\right)^{\beta}}\right]^{b(i+j)}
$$

Using (7), we have

$$
F(x)=\sum_{i, j, k=0}^{\infty}(-1)^{i+j+k} p^{i}\left(\begin{array}{c}
-a \\
i
\end{array}\right)\left(\begin{array}{c}
a \\
j
\end{array}\right)\left(\begin{array}{c}
b(i+j) \\
k
\end{array}\right) \mathrm{e}^{-k\left(\frac{\alpha}{x}\right)^{\beta}} .
$$

Then, the cdf of the EMOFr reduces to

$$
F(x)=\sum_{k=0}^{\infty} v_{k} G\left(x ; \alpha k^{1 / \beta}, \beta\right),
$$


where

$$
v_{k}=\sum_{i, j=0}^{\infty}(-1)^{i+j+k} p^{i}\left(\begin{array}{c}
-a \\
i
\end{array}\right)\left(\begin{array}{c}
a \\
j
\end{array}\right)\left(\begin{array}{c}
b(i+j) \\
k
\end{array}\right)
$$

and $G\left(x ; \alpha k^{1 / \beta}, \beta\right)$ is the cdf of the Fréchet distribution with scale parameter $\alpha k^{1 / \beta}$ and shape parameter $\beta$.

By integrating equation (8), we obtain

$$
f(x)=\sum_{k=0}^{\infty} v_{k} g\left(x ; \alpha k^{1 / \beta}, \beta\right),
$$

where $g\left(x ; \alpha k^{1 / \beta}, \beta\right)$ is the Fréchet density with scale parameter $\alpha k^{1 / \beta}$ and shape parameter $\beta$. Equation (9) reveals that the EMOFr density can be written as a linear combination of Fréchet densities. So, several of its mathematical properties can be obtained from those of the Fréchet distribution and equation (9).

\section{Mathematical Properties}

In this section, we derive some mathematical properties of the EMOFr distribution including ordinary and incomplete moments, quantile and generating functions and order statistics.

Let $Z$ be a random variable having the Fréchet distribution defined in Section 2. For $r<$ $\beta$, the $n$th ordinary and incomplete moments of $Z$ are given by

$$
\mu_{n}^{\prime}=\alpha^{n} \Gamma(1-n / \beta) \text { and } \varphi_{n, Z}(t)=\alpha^{n} \gamma\left(1-n / \beta,(\alpha / t)^{\beta}\right),
$$

respectively, where $\Gamma(a)=\int_{0}^{\infty} y^{a-1} e^{-y} d y$ is the complete gamma function and $\gamma(a, z)=\int_{0}^{z} y^{a-1} e^{-y} d y$ is the lower incomplete gamma function.

\subsection{Ordinary and Incomplete Moments}

The $n$th ordinary moment of $X$ is given by

$$
\mu_{n}^{\prime}=E\left(X^{n}\right)=\sum_{k=0}^{\infty} v_{k} \int_{0}^{\infty} x^{n} g\left(x ; \alpha k^{1 / \beta}, \beta\right) d x .
$$

For $n<\beta$, we obtain

$$
\mu_{n}^{\prime}=\sum_{k=0}^{\infty} v_{k} \alpha^{n} k^{n / \beta} \Gamma\left(1-\frac{n}{\beta}\right) .
$$

The mean of $X$ follows from the last equation with $n=1$, that is,

$$
\mu_{1}^{\prime}=\sum_{k=0}^{\infty} v_{k} \alpha k^{1 / \beta} \Gamma(1-1 / \beta)
$$

The skewness and kurtosis measures can be evaluated from the ordinary moments using well-known relationships. 
The $n$th incomplete moment is defined by

$$
\varphi_{n}(t)=\int_{0}^{t} x^{s} f(x) d x
$$

Using equation (9), we can write

$$
\varphi_{s}(t)={ }_{k=0}^{\infty} v_{k} \int_{0}^{t} x^{n} g\left(x ; \alpha k^{1 / \beta}, \beta\right) d x .
$$

Then, the $n$th incomplete moment of the EMOFr distribution is given (for $n<\beta$ ) by

$$
\varphi_{n}(t)={ }_{k=0}^{\infty} v_{k} \alpha^{n} k^{n / \beta} \gamma\left(1-\frac{n}{\beta}, k\left(\frac{\alpha}{t}\right)^{\beta}\right) .
$$

The first incomplete moment, denoted by $\varphi_{1}(t)$, is

$$
\varphi_{1}(t)={ }_{k=0}^{\infty} v_{k} \alpha k^{1 / \beta} \gamma\left(1-\frac{1}{\beta}, k\left(\frac{\alpha}{t}\right)^{\beta}\right)
$$

and it has an important applications related to the Bonferroni and Lorenz curves and the mean residual life (MRL) and the mean inactivity time (MIT). The Bonferroni and Lorenz curves are very useful in economics, demography, insurance, engineering and medicine. The MRL and MIT are defined by $m_{1}(t)=\left[1-\varphi_{1}(t)\right] / R(t)-t$ and $M_{1}(t)=t-\varphi_{1}(t) / F(t)$, respectively.

\subsection{Quantile and Generating Functions}

The quantile function of $X$ is determined by inverting (3) as

$$
Q(u)=\alpha\left\{-\log \left[1-\left(\frac{1-u^{\frac{1}{a}}}{1-p u^{\frac{1}{a}}}\right)^{\frac{1}{b}}\right]\right\}^{-1 / \beta} \quad, 0<u<1 .
$$

Simulating the EMOFr random variable is straightforward. If $U$ is a uniform variate in the unit interval $(0,1)$, the random variable $X=Q(U)$ follows (4).

Now, we introduce the moment generating function (mgf) of the Fréchet distribution defined in Section 2, denoted by $M(t ; \alpha, \beta)$, as derived in Afify et al. (2016b). Based on $M(t ; \alpha, \beta)$, we will derive the mgf of the EMOFr distribution.

$$
M(t ; \alpha, \beta)=E[\exp (t x)]=\int_{0}^{\infty} \exp (t x) g(x) d x .
$$

Let $y=1 / x$, we have

$$
M(t ; \alpha, \beta)=\beta \alpha^{\beta} \int_{0}^{\infty} \exp \left(\frac{t}{y}\right) y^{\beta-1} \exp \left[-(\alpha y)^{\beta}\right] d y .
$$

Using the exponential series for $\exp \left(\frac{t}{y}\right)$, we can write

$$
\exp \left(\frac{t}{y}\right)=\sum_{m=0}^{\infty} \frac{t^{m}}{m !} y^{-m}
$$


Then, we obtain

$$
M(t ; \alpha, \beta)=\beta \alpha^{\beta} \sum_{m=0}^{\infty} \frac{t^{m}}{m !} \int_{0}^{\infty} y^{\beta-m-1} \exp \left[-(\alpha y)^{\beta}\right] d y .
$$

After some algebra, we can write

$$
M(t ; \alpha, \beta)=\sum_{m=0}^{\infty} \frac{\alpha^{m} t^{m}}{m !} \Gamma\left(\frac{\beta-m}{\beta}\right)
$$

Using the Wright generalized hypergeometric function defined by

$$
{ }_{p} \Psi_{q}\left[\begin{array}{l}
\left(\alpha_{1}, A_{1}\right), \ldots,\left(\alpha_{p}, A_{p}\right) \\
\left(\beta_{1}, B_{1}\right), \ldots,\left(\beta_{q}, B_{q}\right)
\end{array} ; x\right]=\sum_{n=0}^{\infty} \frac{\prod_{j=1}^{p} \Gamma\left(\alpha_{j}+A_{j} n\right)}{\prod_{j=1}^{q} \Gamma\left(\beta_{j}+B_{j} n\right)} \frac{x^{n}}{n !} .
$$

Thus, the mgf of the Fréchet distribution reduces to

$$
M(t ; \alpha, \beta)={ }_{1} \Psi_{0}\left[\left(1,-\beta^{-1}\right) ; \alpha t\right] .
$$

Based on equations (9) and (10), the mgf of $X$, say $M(t)$, is given by

$$
M(t)=\sum_{k=0}^{\infty} v_{k 1} \Psi_{0}\left[\left(1,-\beta^{-1}\right) ; \alpha k^{1 / \beta} t\right]
$$

\subsection{Order Statistics}

Let $X_{1}, \ldots, X_{n}$ be a random sample of size $n$ from the EMOFr distribution and $X_{(1)}, \ldots, X_{(n)}$ be the corresponding order statistics. Then, the pdf of the $i$ th order statistic $X_{i: n}$, say $f_{i: n}(x)$, is given by

$$
f_{i: n}(x)=\frac{f(x)}{\mathrm{B}(i, n-i+1)} \sum_{j=0}^{n-i}(-1)^{j}\left(\begin{array}{l}
n-i \\
j
\end{array}\right) F(x)^{i+j-1}
$$

Using equations (3) and (4), we can write

$$
\begin{aligned}
& f(x) F(x)^{i+j-1}=a b(1-p) \beta \alpha^{\beta} x^{-\beta-1} \mathrm{e}^{-\left(\frac{\alpha}{x}\right)^{\beta}}\left[1-\mathrm{e}^{-\left(\frac{\alpha}{x}\right)^{\beta}}\right]^{b-1} \\
& \times\left\{1-\left[1-\mathrm{e}^{-\left(\frac{\alpha}{x}\right)^{\beta}}\right]^{b}\right\}^{a(i+j)-1}\left\{1-p\left[1-\mathrm{e}^{-\left(\frac{\alpha}{x}\right)^{\beta}}\right]^{b}\right\}^{-a(i+j)-1}
\end{aligned}
$$

Applying the expansions (5) and (7), and after some simplifications, we have

$$
\begin{aligned}
& f(x) F(x)^{i+j-1}=a b(1-p) \beta \alpha^{\beta} x^{-\beta-1} \sum_{r, w, k=0}^{\infty}(-1)^{r+w+k} p^{r} \\
& \times\left(\begin{array}{c}
-a(i+j)-1 \\
r
\end{array}\right)\left(\begin{array}{c}
a(i+j)-1 \\
w
\end{array}\right)\left(\begin{array}{c}
b(r+w+1)-1 \\
k
\end{array}\right) \mathrm{e}^{-(k+1)\left(\frac{\alpha}{x}\right)^{\beta}} .
\end{aligned}
$$

By inserting the last equation in equation (11), we obtain

$$
f_{i: n}(x)=\sum_{k=0}^{\infty} \eta_{k} g\left(x ; \alpha(k+1)^{1 / \beta}, \beta\right),
$$


where $g\left(x ; \alpha(k+1)^{1 / \beta}, \beta\right)$ denotes the pdf of the Fréchet distribution with parameters $\alpha(k+1)^{1 / \beta}$ and $\beta$ and

$$
\begin{gathered}
\eta_{k}=\sum_{r, w=0}^{\infty} \sum_{j=0}^{n-i} \frac{(-1)^{j+r+w+k} p^{r} a b(1-p)}{B(i, n-i+1)(k+1)}\left(\begin{array}{c}
n-i \\
j
\end{array}\right)\left(\begin{array}{c}
-a(i+j)-1 \\
r
\end{array}\right) \\
\times\left(\begin{array}{c}
a(i+j)-1 \\
w
\end{array}\right)\left(\begin{array}{c}
b(r+w+1)-1 \\
k
\end{array}\right) .
\end{gathered}
$$

Equation (12) means that the pdf of the EMOFr order statistics is a linear mixture of Fréchet densities.

The $q$ th moment of $X_{i: n}$ (for $q<\alpha$ ) is given by

$$
E\left(X_{i: n}^{q}\right)=\sum_{k=0}^{\infty} \eta_{k} \alpha^{q}(k+1)^{q / \beta} \Gamma\left(1-\frac{q}{\beta}\right) .
$$

\section{Estimation}

In this section, we consider the estimation of the unknown parameters of the EMOFr from complete samples only by maximum likelihood. We investigate the MLEs of the parameters of the $\operatorname{EMOFr}(\alpha, \beta, a, b, p)$ model. Let $\mathbf{x}=\left(x_{1}, \ldots, x_{n}\right)$ be a random sample from this model with unknown parameter vector $v=(\alpha, \beta, a, b, p)^{T}$.

The log-likelihood function for $\theta$, say $\ell=\ell(\theta)$, is given by

$$
\begin{gathered}
\ell=n(\log a+\log b+\log (1-p)+\log (\beta)+\beta \log (\alpha))-\sum_{i=1}^{n}\left(\frac{\alpha}{x_{i}}\right)^{\beta} \\
+(b-1) \sum_{i=1}^{n} \log \left(1-s_{i}\right)+(a-1) \sum_{i=1}^{n} \log \left[1-\left(1-s_{i}\right)^{b}\right] \\
-(\beta+1) \sum_{i=1}^{n} \log x_{i}-(a+1) \sum_{i=1}^{n} \log \left[1-p\left(1-s_{i}\right)^{b}\right],
\end{gathered}
$$

where $s_{i}=\mathrm{e}^{-\left(\frac{\alpha}{x_{i}}\right)^{\beta}}$

The above equation can be maximized either directly by using the R (optim function), SAS (PROC NLMIXED sub-routine), Ox program (MaxBFGS) or by solving the nonlinear likelihood equations obtained by differentiating it.

The score vector is given by $\mathbf{U}(\theta)=\frac{\partial \ell}{\partial \theta}=\left(\frac{\partial \ell}{\partial \alpha}, \frac{\partial \ell}{\partial \beta}, \frac{\partial \ell}{\partial a}, \frac{\partial \ell}{\partial b}, \frac{\partial \ell}{\partial p}\right)^{T}$. Then, we have

$$
\begin{aligned}
& \frac{\partial \ell}{\partial \alpha}=\frac{n \beta}{\alpha}-\frac{\beta}{\alpha} \sum_{i=1}^{n}\left(\frac{\alpha}{x_{i}}\right)^{\beta}+\frac{\beta(b-1)}{\alpha} \sum_{i=1}^{n} \frac{s_{i}\left(\frac{\alpha}{x_{i}}\right)^{\beta}}{1-s_{i}} \\
& -\frac{\beta b(a-1)}{\alpha} \sum_{i=1}^{n} \frac{\left(\frac{\alpha}{x_{i}}\right)^{\beta} s_{i}\left(1-s_{i}\right)^{b-1}}{1-\left(1-s_{i}\right)^{b}}+\frac{\beta b p(a+1)}{\alpha} \sum_{i=1}^{n} \frac{s_{i}\left(\frac{\alpha}{x_{i}}\right)^{\beta}\left(1-s_{i}\right)^{b-1}}{1-p\left(1-s_{i}\right)^{b}}
\end{aligned}
$$




$$
\begin{aligned}
& \frac{\partial \ell}{\partial \beta}=\frac{n}{\beta}+n \log \alpha-\sum_{i=1}^{n} \log x_{i}-\sum_{i=1}^{n}\left(\frac{\alpha}{x_{i}}\right)^{\beta} \log \left(\frac{\alpha}{x_{i}}\right) \\
& (b-1) \sum_{i=1}^{n} \frac{\left(\frac{\alpha}{x_{i}}\right)^{\beta} \mathrm{e}^{-\left(\frac{\alpha}{x_{i}}\right)^{\beta}} \log \left(\frac{\alpha}{x_{i}}\right)}{1-\mathrm{e}^{-\left(\frac{\alpha}{x_{i}}\right)^{\beta}}} \\
& -b(a-1) \sum_{i=1}^{n} \frac{s_{i}\left(\frac{\alpha}{x_{i}}\right)^{\beta}\left(1-s_{i}\right)^{b-1} \log \left(\frac{\alpha}{x_{i}}\right)}{1-\left(1-s_{i}\right)^{b}} \\
& +b p(a+1) \sum_{i=1}^{n} \frac{s_{i}\left(\frac{\alpha}{x_{i}}\right)^{\beta}\left(1-s_{i}\right)^{b-1} \log \left(\frac{\alpha}{x_{i}}\right)}{1-p\left(1-s_{i}\right)^{b}} \\
& \frac{\partial \ell}{\partial a}=\frac{n}{a}+\sum_{i=1}^{n} \log \left[1-\left(1-s_{i}\right)^{b}\right]-\sum_{i=1}^{n} \log \left[1-p\left(1-s_{i}\right)^{b}\right] \\
& \frac{\partial \ell}{\partial b}=\frac{n}{b}+\sum_{i=1}^{n} \log \left(1-s_{i}\right)-(a-1) \sum_{i=1}^{n} \frac{\left(1-s_{i}\right)^{b} \log \left(1-s_{i}\right)}{1-\left(1-s_{i}\right)^{b}} \\
& +p(a+1) \sum_{i=1}^{n} \frac{\left(1-s_{i}\right)^{b} \log \left(1-s_{i}\right)}{1-p\left(1-s_{i}\right)^{b}}
\end{aligned}
$$

and

$$
\frac{\partial \ell}{\partial p}=\frac{-n}{1-p}+(a+1) \sum_{i=1}^{n} \frac{\left(1-s_{i}\right)^{b}}{1-p\left(1-s_{i}\right)^{b}}
$$

We can obtain the estimates of the unknown parameters by setting the score vector to zero, $\mathbf{U}(\hat{\theta})=\mathbf{0}$. By solving these equations simultaneously gives the MLEs $\hat{\alpha}, \hat{\beta}, \hat{a}, \hat{b}$ and $\hat{p}$. These estimates can be obtained numerically using iterative techniques such as the Newton-Raphson algorithm. For the EMOFr distribution, all the second-order derivatives exist.

For interval estimation of the model parameters, we require the $5 \times 5$ observed information matrix $J(\theta)=\left\{J_{r s}\right\}$ for $r, s=\alpha, \beta, a, b, p$ Under standard regularity conditions, the multivariate normal $N_{5}\left(0, J(\hat{\theta})^{-1}\right)$ distribution can be used to construct approximate confidence intervals for the model parameters. Here, $J(\hat{\theta})$ is the total observed information matrix evaluated at $\hat{\theta}$. Then, approximate $100(1-\phi) \%$ confidence intervals for the model parameters can be determined in the usual way of the first-order asymptotic theory. 


\section{Applications}

This section is devoted to illustrate the importance of the EMOFr distribution empirically using two applications to real data sets. The first data set refers to the survival times, in weeks, of 33 patients suffering from acute Myelogenous Leukemia (Feigl and Zelen, 1965). The second data set represents the exceedances of flood peaks (in $\mathrm{m}^{3} / \mathrm{s}$ ) of the Wheaton River near Carcross in Yukon Territory, Canada.

For the two data sets, we shall compare the fit of the EMOFr distribution with the Kumaraswamy Marshall-Olkin Fréchet (KMOFr), Kumaraswamy Fréchet (KFr), exponentiated Fréchet (EFr), gamma extended Fréchet (GEFr), beta Fréchet (BFr), beta exponential Fréchet (BExFr), transmuted Fréchet (TFr) and Fréchet (Fr) distributions with corresponding pdfs (for $x>0$ ):

KMOFr: $f(x)=\alpha \beta a b \delta^{\beta} x^{-\beta-1} \exp \left[-a\left(\frac{\delta}{x}\right)^{\beta}\right]\left\{\alpha+(1-\alpha) \exp \left[-\left(\frac{\delta}{x}\right)^{\beta}\right]\right\}^{-a-1}$

$$
\times\left\{1-\exp \left[-a\left(\frac{\delta}{x}\right)^{\beta}\right]\left\{\alpha+(1-\alpha) \exp \left[-\left(\frac{\delta}{x}\right)^{\beta}\right]\right\}^{-a}\right\}^{b-1}
$$

KFr: $f(x)=a b \beta \alpha^{\beta} x^{-(\beta+1)} \exp \left[-a\left(\frac{\alpha}{x}\right)^{\beta}\right]\left\{1-\exp \left[-a\left(\frac{\alpha}{x}\right)^{\beta}\right]\right\}^{b-1}$;

EFr: $f(x)=\theta \beta \alpha^{\beta} x^{-(\beta+1)} \exp \left[-\left(\frac{\alpha}{x}\right)^{\beta}\right]\left\{1-\exp \left[-\left(\frac{\alpha}{x}\right)^{\beta}\right]\right\}^{\theta-1}$;

GEFr: $f(x)=\frac{a \beta \alpha^{\beta}}{\Gamma(b)} x^{-(\beta+1)} \exp \left[-\left(\frac{\alpha}{x}\right)^{\beta}\right]\left\{1-\exp \left[-\left(\frac{\alpha}{x}\right)^{\beta}\right]\right\}^{a-1}$

$$
\times\left\{-\log \left\{1-\exp \left[-\left(\frac{\alpha}{x}\right)^{\beta}\right]\right\}^{a}\right\}^{b-1} \text {; }
$$

BFr: $f(x)=\frac{\beta \alpha^{\beta}}{B(a, b)} x^{-(\beta+1)} \exp \left[-a\left(\frac{\alpha}{x}\right)^{\beta}\right]\left\{1-\exp \left[-\left(\frac{\alpha}{x}\right)^{\beta}\right]\right\}^{b-1}$;

BExFr: $f(x)=\frac{\lambda \beta \alpha^{\beta}}{B(a, b)} x^{-\beta-1} \exp \left[-\left(\frac{\alpha}{x}\right)^{\beta}\right]\left\{-\exp \left[-\left(\frac{\alpha}{x}\right)^{\beta}\right]\right\}^{\lambda b-1}$

$$
\times\left(1-\left\{1-\exp \left[-\left(\frac{\alpha}{x}\right)^{\beta}\right]\right\}^{\lambda}\right)^{a-1}
$$

TFr: $f(x)=\beta \alpha^{\beta} x^{-\beta-1} \exp \left[-\left(\frac{\alpha}{x}\right)^{\beta}\right]\left\{\lambda+1-2 \lambda \exp \left[-\left(\frac{\alpha}{x}\right)^{\beta}\right]\right\}$.

The parameters of the above densities are all positive real numbers except for the TFr distribution for which $|\lambda| \leq 1$.

The fitted distributions are compared using the following criteria: the $-2 \hat{\ell}$ (Maximized Log-Likelihood), AIC (Akaike Information Criterion), CAIC (Consistent Akaike 
Information Criterion), BIC (Bayesian Information Criterion) and HQIC (Hannan-Quinn Information Criterion).

Table 2: Goodness-of-fit statistics for Leukemia data

\begin{tabular}{|l|c|c|c|c|c|c|c|}
\hline Model & $-2 \hat{\ell}$ & $A I C$ & CAIC & HQIC & BIC & $W^{*}$ & $A^{*}$ \\
\hline EMOFr & 299.122 & 309.122 & 311.344 & 311.639 & 316.605 & 0.03552 & 0.26312 \\
\hline KMOFr & 304.804 & 314.804 & 317.026 & 317.321 & 322.286 & 0.08367 & 0.54694 \\
\hline KFr & 304.832 & 314.832 & 316.261 & 316.846 & 320.818 & 0.09461 & 0.63420 \\
\hline EFr & 307.788 & 313.788 & 314.616 & 315.299 & 318.277 & 0.11151 & 0.70509 \\
\hline GEFr & 307.861 & 315.861 & 317.289 & 317.875 & 321.847 & 0.11385 & 0.71476 \\
\hline BFr & 307.991 & 315.991 & 317.420 & 318.006 & 321.978 & 0.11569 & 0.72387 \\
\hline BExFr & 309.905 & 319.905 & 322.127 & 322.422 & 327.387 & 0.13931 & 0.85497 \\
\hline TFr & 311.449 & 317.449 & 318.276 & 318.959 & 321.938 & 0.15502 & 0.94183 \\
\hline Fr & 311.997 & 315.997 & 316.397 & 317.004 & 318.990 & 0.16011 & 0.97592 \\
\hline
\end{tabular}

Table 3: MLEs and their standard errors (in parentheses) for Leukemia data

\begin{tabular}{|l|c|c|c|c|c|}
\hline Model & \multicolumn{5}{|c|}{ Estimates } \\
\hline Fr & $\begin{array}{c}7.8652 \\
(2.0913)\end{array}$ & $\begin{array}{c}0.6944 \\
(0.0915)\end{array}$ & & & \\
\hline $\operatorname{TFr}$ & 5.5489 & 0.7401 & -0.4291 & & \\
$(\alpha, \beta, \lambda)$ & $(2.9837)$ & $(0.0995)$ & $(0.5549)$ & & \\
\hline $\mathrm{EFr}$ & 1426.6289 & 0.24909 & 13.7467 & & \\
$(\alpha, \beta, \theta)$ & $(3607.173)$ & $(0.0708)$ & $(13.5121)$ & & \\
\hline $\mathrm{KFr}$ & 9378.570 & 0.0842 & 5.5132 & 7160.57 & \\
$(\alpha, \beta, a, b)$ & $(804.3827)$ & $(0.0247)$ & $(2.2439)$ & $(17494.23)$ & \\
\hline $\mathrm{BFr}$ & 24.2231 & 0.0884 & 33.5337 & 60.5680 & \\
$(\alpha, \beta, a, b)$ & $(305.1054)$ & $(0.119015)$ & $(111.4965)$ & $(161.2934)$ & \\
\hline $\mathrm{GEFr}$ & 62.7173 & 0.0555 & 189.5528 & 79.3368 & \\
$(\alpha, \beta, a, b)$ & $(1756.0625)$ & $(0.1070)$ & $(738.3019)$ & $(388.7273)$ & \\
\hline $\mathrm{EMOFr}$ & 70.9780 & 0.7831 & 0.12661 & 6.6523 & -110.2490 \\
$(\alpha, \beta, a, b, p)$ & $(0.1027)$ & $(0.0925)$ & $(0.0419)$ & $(0.2838)$ & $(98.8912)$ \\
\hline $\mathrm{KMOFr}$ & 31946.73 & 0.6074 & 4.8067 & 1.0146 & 13724.51 \\
$(\alpha, \beta, \delta, a, b)$ & $(579.3846)$ & $(0.1067)$ & $(6.1015)$ & $(0.1390)$ & $(10687.72)$ \\
\hline $\mathrm{BExFr}$ & 0.1162 & 4.3641 & 0.0437 & 9.3849 & 6.4056 \\
$(\alpha, \beta, \lambda, a, b)$ & $(0.0265)$ & $(0.0253)$ & $(0.0489)$ & $(2.7092)$ & $(9.6967)$ \\
\hline
\end{tabular}




\section{The Exponentiated Marshall-Olkin Fréchet Distribution}

Tables 2 and 4 provide the numerical values of goodness-of-fit statistics or the fitted models, whereas the values of the MLEs and their corresponding standard errors (in parentheses) of the model parameters are listed in Tables 3 and 5, respectively.

The plots of fitted densities for the EMOFr model and other models, for both data sets, are displayed in Figures 3 and 6. Figures 4 and 7 display the QQ plots for both data sets, respectively. The estimated cdfs, for both data sets, of the competitive models are given in Figures 5 and 8.
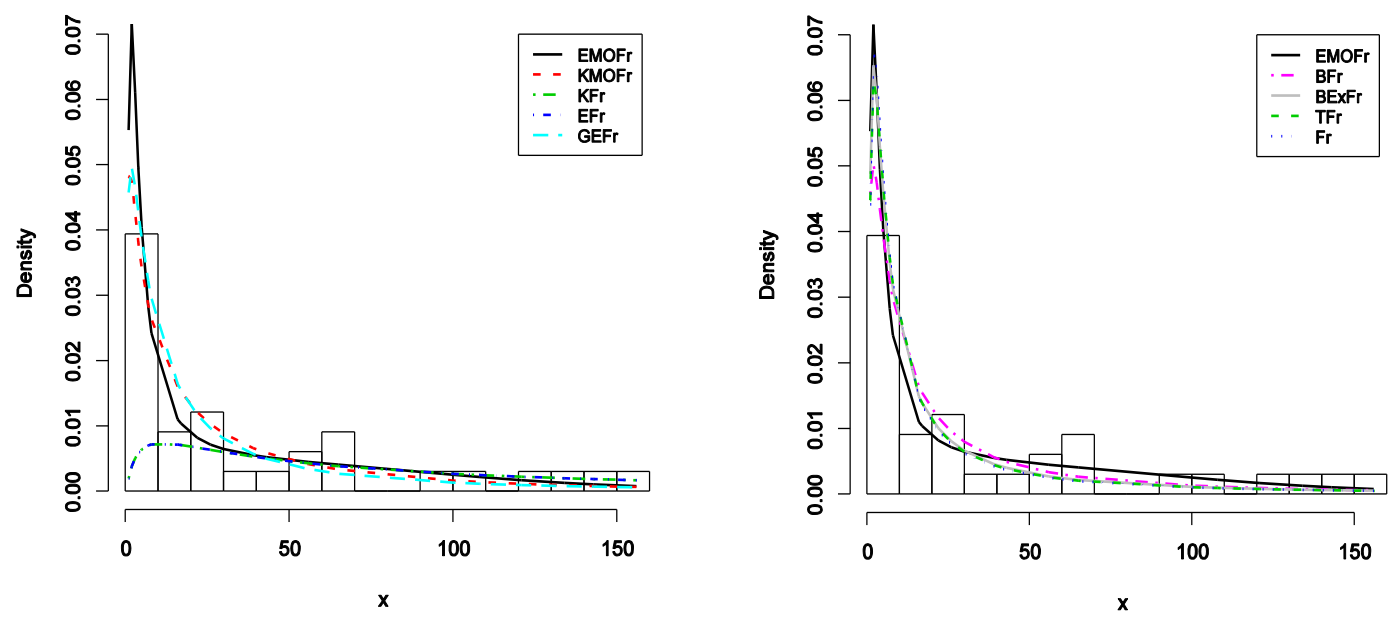

Figure 3: The fitted pdfs of the EMOFr model and other models for Leukemia data
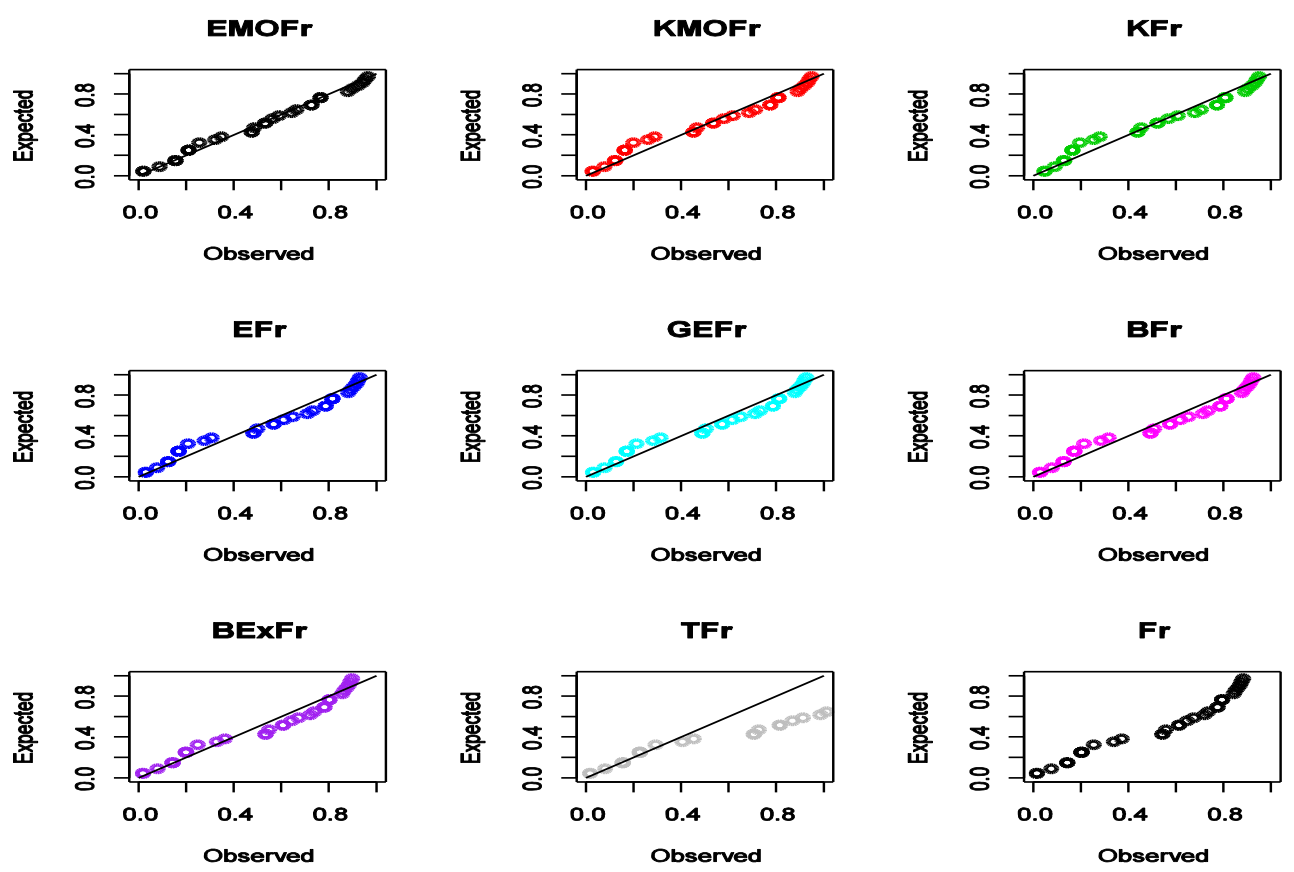

Figure 4: Q-Q plots for Leukemia data 

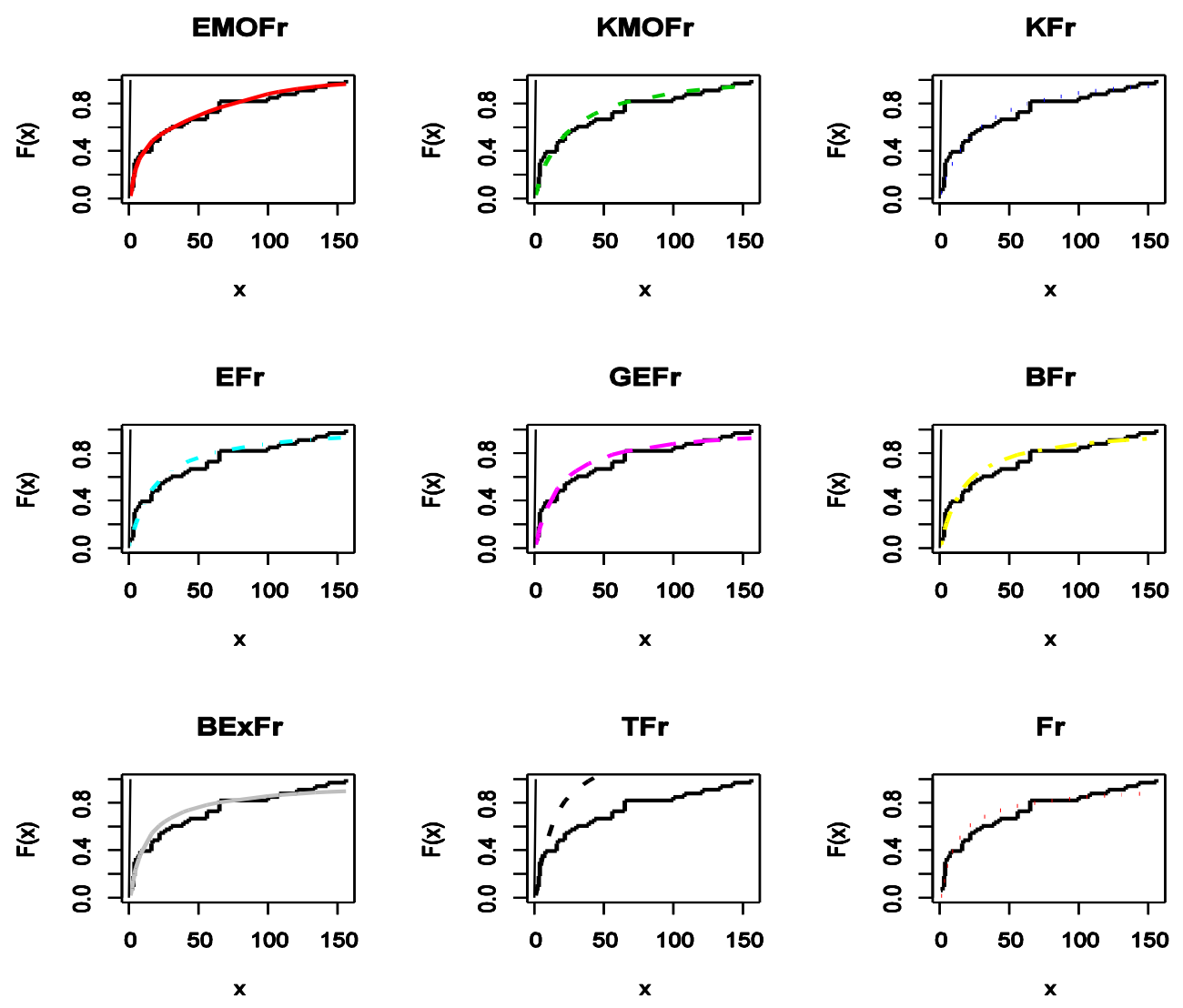

Figure 5: Fitted cdfs on empirical cdf for Leukemia data

Tables 2 and 4 compare the EMOFr model with the KMOFr, KFr, EFr, GEFr, BFr, $\mathrm{BExFr}$, TFr and Fr distributions. The proposed model has the lowest values for all goodness-of-fit statistics among all fitted models. Thus, the EMOFr model can be chosen as the best model to fit both data sets. The plots in the Figures 3 through 8 prove that the EMOFr distribution gives a better fit than other nested and non-nested models for both data sets.

Table 4: Goodness-of-fit statistics for Wheaton River data

\begin{tabular}{|l|c|c|c|c|c|c|c|}
\hline Model & $-2 \ell$ & $A I C$ & $C A I C$ & $H Q I C$ & $B I C$ & $W^{*}$ & $A^{*}$ \\
\hline EMOFr & 495.369 & 505.369 & 506.278 & 509.901 & 516.753 & 0.04419 & 0.26324 \\
\hline KMOFr & 502.154 & 512.154 & 513.063 & 516.686 & 523.537 & 0.13817 & 0.76472 \\
\hline KFr & 506.005 & 514.005 & 514.602 & 517.630 & 523.112 & 0.17337 & 0.97379 \\
\hline EFr & 512.243 & 518.243 & 518.596 & 520.962 & 525.073 & 0.24225 & 1.37968 \\
\hline GEFr & 514.651 & 522.651 & 523.248 & 526.277 & 531.758 & 0.28449 & 1.60447 \\
\hline BFr & 514.765 & 522.765 & 523.362 & 526.39 & 531.872 & 0.28585 & 1.61240 \\
\hline BExFr & 521.991 & 531.991 & 532.901 & 536.523 & 543.375 & 0.33941 & 1.96148 \\
\hline TFr & 529.984 & 535.984 & 536.337 & 538.703 & 542.814 & 0.41857 & 2.42652 \\
\hline Fr & 534.038 & 538.038 & 538.212 & 539.851 & 542.591 & 0.48147 & 2.80181 \\
\hline
\end{tabular}


Table 5: MLEs and the corresponding SEs (in parentheses) for Wheaton River data

\begin{tabular}{|l|c|c|c|c|c|}
\hline Model & \multicolumn{5}{|c|}{ Estimates } \\
\hline Fr & 2.879 & 0.6521 & & & \\
\hline$(\alpha, \beta)$ & $(0.553)$ & $(0.054)$ & & & \\
\hline $\mathrm{TFr}$ & 1.5083 & 0.7107 & -0.7289 & & \\
\hline$(\alpha, \beta, \lambda)$ & $(0.4374)$ & $(0.0589)$ & $(0.2338)$ & & \\
\hline $\mathrm{EFr}$ & 391.9297 & 0.2677 & 14.4425 & & \\
\hline$(\alpha, \beta, \theta)$ & $(398.185)$ & $(0.033)$ & $(6.62)$ & & \\
\hline $\mathrm{KFr}$ & 6.3401 & 0.1332 & 6.6065 & 478.3001 & \\
\hline$(\alpha, \beta, a, b)$ & $(0.011)$ & $(1.677 .10-4)$ & $(0.011)$ & $(0.132)$ & \\
\hline $\mathrm{BFr}$ & 38.2262 & 0.1356 & 11.712 & 30.3168 & \\
\hline$(\alpha, \beta, a, b)$ & $(118.541)$ & $(0.082)$ & $(20.38)$ & $(34.144)$ & \\
\hline $\mathrm{GEFr}$ & 40.4813 & 0.1345 & 35.7391 & 11.7358 & \\
\hline$(\alpha, \beta, a, b)$ & $(129.175)$ & $(0.081)$ & $(42.978)$ & $(20.235)$ & \\
\hline $\mathrm{EMOFr}$ & 284.0069 & 0.4225 & 0.2157 & 40.2609 & -11.1025 \\
\hline$(\alpha, \beta, a, b, p)$ & $(0.1214)$ & $(0.1033)$ & $(0.1428)$ & $(25.8295)$ & $(10.1829)$ \\
\hline $\mathrm{KMOFr}$ & 89263.64 & 0.6814 & 0.2556 & 1.2323 & 54816.88 \\
\hline$(\alpha, \beta, \delta, a, b)$ & $(224.9697)$ & $(0.0619)$ & $(0.4076)$ & $(0.1503)$ & $(15004.53)$ \\
\hline $\mathrm{BExFr}$ & 0.0068 & 1.8401 & 0.0494 & 18.8169 & 22.5355 \\
\hline$(\alpha, \beta, \lambda, a, b)$ & $(0.0044)$ & $(0.7017)$ & $(0.0356)$ & $(4.9244)$ & $(20.6856)$ \\
\hline
\end{tabular}
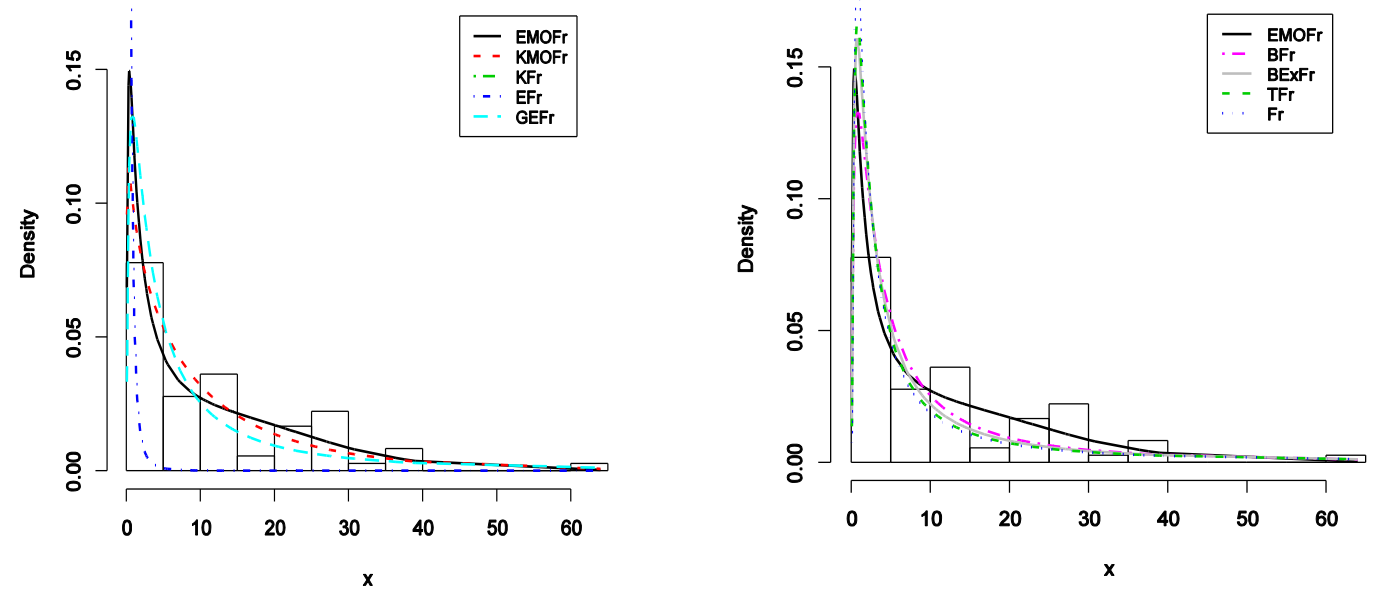

Figure 6: The fitted pdfs of the EMOFr model and other models for Wheaton River data 


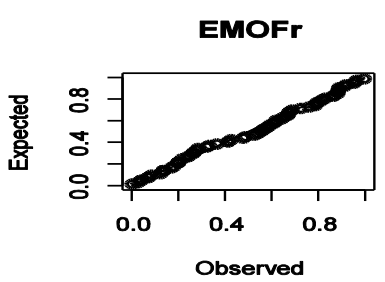

EFr

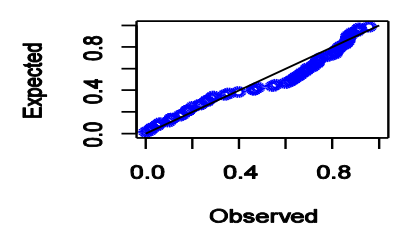

BEXFr

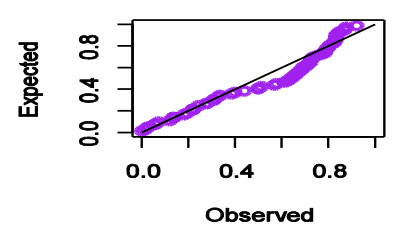

KMOFr

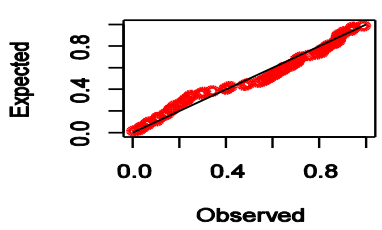

GEFr

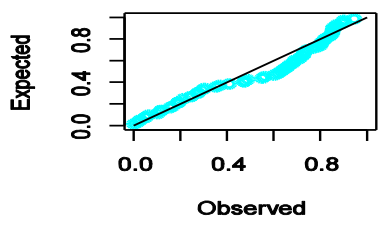

TFr

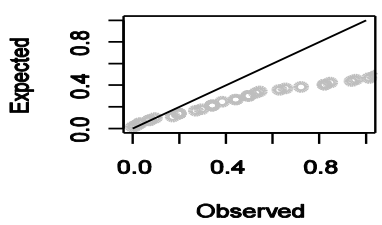

KFr

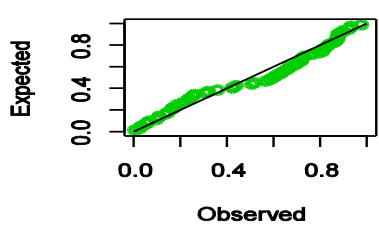

BFr

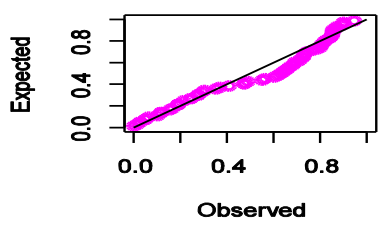

Fr

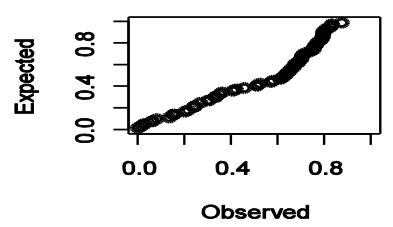

Figure 7: Q-Q plots for Wheaton River data
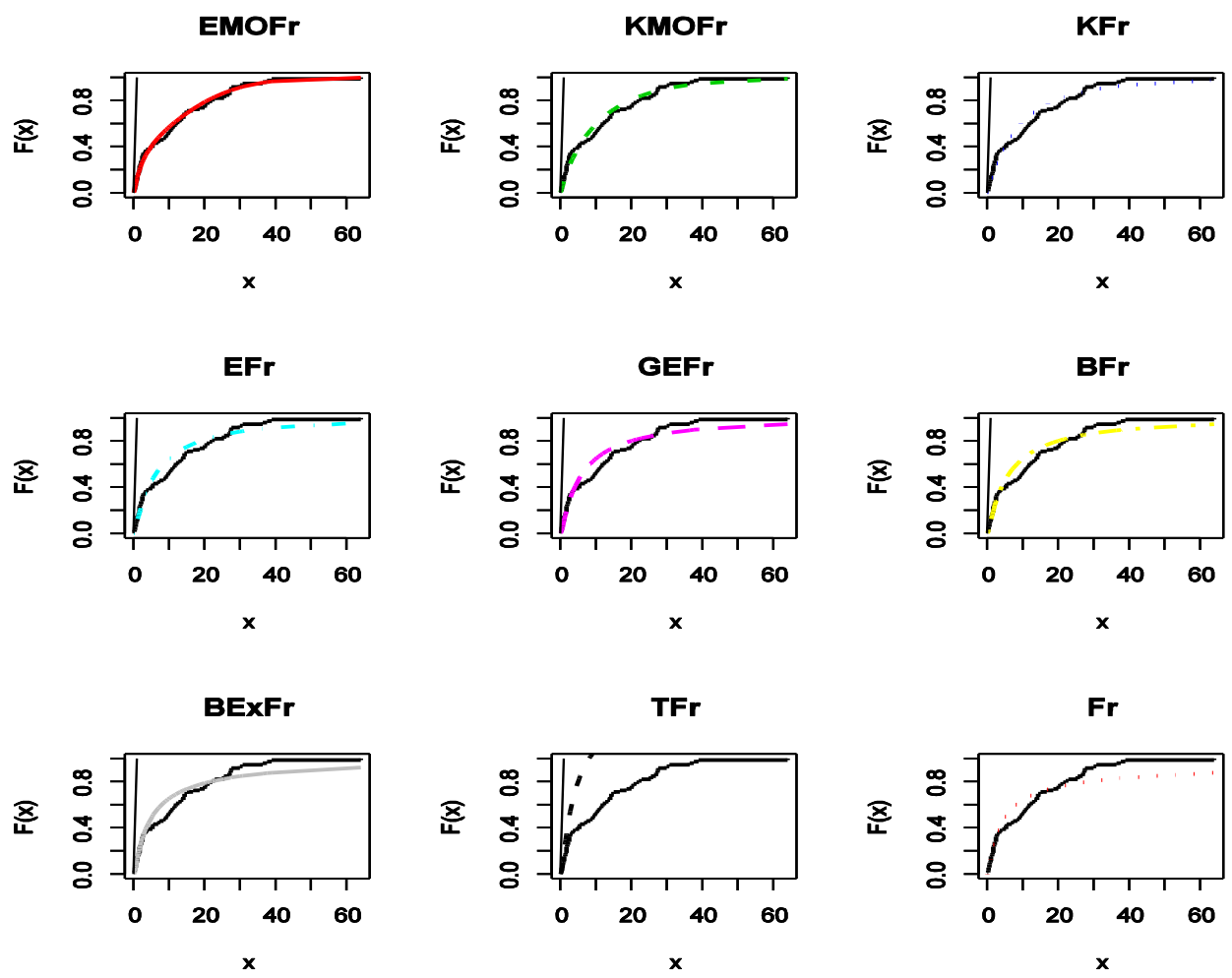

Figure 8: Fitted cdfs on empirical cdf for Wheaton River data 


\section{Conclusions}

In this paper, we study a new five-parameter model named the exponentiated MarshallOlkin Fréchet (EMOFr) distribution, which extends the Fréchet and Marshall-Olkin Fréchet distributions. The EMOFr density function is a linear mixture of Fréchet densities. We derive explicit expressions for its mathematical properties including the ordinary and incomplete moments, quantile and generating function and order statistics. The model parameters are estimated by maximum likelihood. The new distribution applied to two real data sets provides better fits than some other competitive models.

\section{References}

1. Afify, A. Z., Hamedani, G. G., Ghosh, I. and Mead, M. E. (2015). The transmuted Marshall-Olkin Fréchet distribution: properties and applications. International Journal of Statistics and Probability, 4, 132-184.

2. Afify, A. Z., Yousof, H. M., Cordeiro, G. M., Nofal, Z. M. and Ahmed, A. N. (2016a). The Kumaraswamy Marshall-Olkin Fréchet distribution with applications. Journal of ISOSS, 2, 41-58.

3. Afify, A. Z., Yousof, H. M., Cordeiro, G. M. Ortega, E. M. M. and Nofal, Z. M. (2016b) The Weibull Fréchet distribution and its applications. Journal of Applied Statistics, 43, 2608-2626.

4. Barreto-Souza, W. M., Cordeiro, G. M. and Simas, A. B. (2011). Some results for beta Fréchet distribution. Communications in Statistics - Theory and Methods, 40, 798-811.

5. Dias, C. R., Cordeiro, G. M., Alizadeh, M., Marinho, P. R. D. and Coêlho, H. F. C. (2016). Exponentiated Marshall-Olkin family of distributions. Journal of Statistical Distributions and Applications, 3(1), p.15.

6. Elbatal, I. Asha, G. and Raja, V. (2014). Transmuted exponentiated Fréchet distribution: properties and applications. Journal of Statistics Applications an Probability, 3, 379-394.

7. Feigl, P. and Zelen, M. (1965). Estimation of Exponential Probabilities with Concomitant Information. Biometrics, 21, 826-838.

8. Fréchet, M. (1924). Sur la Loi des Erreurs d'Observation. Bulletin de la Soci et e Math ematique de Moscou, 33, 5-8.

9. Harlow, D. G. (2002). Applications of the Fréchet distribution function. International Journal of Materials and Product Technology, 17, 482-495.

10. Krishna, E., Jose, K. K., Alice, T. and Ristic, M. M. (2013). The Marshall-Olkin Fréchet distribution. Communications in Statistics-Theory and Methods, 42, 4091-4107.

11. Kotz, S. and Nadarajah, S. (2000). Extreme value distributions: theory and applications. Imperial College Press, London.

12. Mahmoud, M. R. and Mandouh, R. M. (2013). On the transmuted Fréchet distribution. Journal of Applied Sciences Research, 9, 5553-5561. 
13. Mead, M. E. and Abd-Eltawab A. R. (2014). A note on Kumaraswamy Fréchet distribution. Australian Journal of Basic and Applied Sciences, 8, 294-300.

14. Mead, M. E., Afify, A. Z., Hamedani, G. G. and Ghosh, I. (2017). The beta exponential Fréchet distribution with applications. Austrian Journal of Statistics, 46, 41-63.

15. Nadarajah, S. and Gupta, A. K. (2004). The beta Fréchet distribution. Far East Journal of Theoretical Statistics, 14, 15-24.

16. Nadarajah, S. and Kotz, S. (2003). The exponentiated Fréchet distribution. Interstat Electronic Journal, 1-7.

17. Resnick, S. I. (2013). Extreme values, regular variation and point processes. Springer, New York.

18. Silva, R. V. D., de Andrade, T. A., Maciel, D., Campos, R. P., and Cordeiro, G. M. (2013). A new lifetime model: the gamma extended Fréchet distribution. Journal of Statistical Theory and Applications, 12, 39-54.

19. Tablada, C. J. and Cordeiro, G. M. (2017). The modified Fréchet distribution and its properties. Communications in Statistics-Theory and Methods, forthcoming.

20. Zaharim, A., Najid, S. K., Razali, A. M. and Sopian, K. (2009). Analysing Malaysian wind speed data using statistical distribution. In Proceedings of the 4th IASME/WSEAS International conference on energy and environment, Cambridge, UK. 